\title{
Hydroxamate-Based Histone Deacetylase Inhibitors as Potential Mediators to Induce Dentine Regeneration by Human Dental Pulp Cell
}

\section{Indrani Sulistyowati ${ }^{1}$, Teerawat Sukpaita ${ }^{1}$, Chalida Nakalekha Limjeerajarus ${ }^{2}$ and Ruchanee Salingcarnboriboon Ampornaramveth ${ }^{1,3 *}$}

${ }^{1}$ Research Unit on Oral Microbiology and Immunology, Department of Microbiology, Faculty of Dentistry, Chulalongkorn University, Bangkok, Thailand, ${ }^{2}$ Department of Physiology, Faculty of Dentistry, Chulalongkorn University, Bangkok, Thailand, ${ }^{3}$ Department of Microbiology, Faculty of Dentistry, Chulalongkorn University, Bangkok, Thailand

Human dental pulp cells (hDPCs) have shown their plasticity when treated with the hydroxamate-based histone deacetylase (HDAC) inhibitor members, Trichostatin A (TSA), and suberoylanilide hydroxamic acid (SAHA). However, a comparison of their potency to stimulate odontoblast-like differentiation and mineralization has not been reported. The aim of our study was to confirm and compare these TSA and SAHA effects. Primary hDPCs cultured with/without various TSA or SAHA concentrations were evaluated using 3-(4,5-dimethylthiazol-2-yl)-2,5-diphenyl tetrazolium bromide (MTT), ALP activity, alizarin red staining, and scratch wound healing assays. The inhibitory effect of TSA and SAHA on inhibiting the activity of HDAC was evaluated by HDAC activity assay. Odontoblast-related gene expression was determined using RT-qPCR. The MTT assay indicated that TSA or SAHA did not affect hDPC viability. TSA or SAHA treatment-induced odontoblast-like differentiation as evidenced by a significant increase in alkaline phosphatase activity and mineral deposition after $400 \mathrm{nM}$ TSA or $1 \mu \mathrm{M}$ SAHA treatment. A significant increase in nuclear factor I C, kruppel like factor 4, dentin matrix acidic phosphoprotein 1, dentin sialophosphoprotein, collagen type I alpha 1 chain, alkaline phosphatase (ALPL), integrin-binding sialoprotein, bone gamma-carboxyglutamate protein, vascular endothelial growth factor $\mathrm{A}$, and cyclin-dependent kinase inhibitor 1 A gene expression analyzed by RT-qPCR, at 24, $72 \mathrm{~h}$, 7, and 10 days of treatment. The activity of HDAC in hDPCs culture was significantly inhibited after $72 \mathrm{~h}$ TSA and SAHA treatment. The scratch wound healing assay displayed enhanced cell migration at $72 \mathrm{~h}$ after TSA or SAHA treatment. Our findings demonstrated that TSA and SAHA have similar stimulatory effects in inducing HDPC odontogenic differentiation and mineralization and propose another potential use of TSA and SAHA to promote dentin regeneration.

Keywords: trichostatin, suberoylanilide hydroxamic acid, odontoblast-like differentiation, human dental pulp cells, dentine regeneration, hydroxamate HDAC inhibitors 


\section{INTRODUCTION}

The breakdown of the dentine layer triggered by a deep dental cavity commonly leads to dental pulp inflammation (pulpitis) (1), and a pulp-capping material may be required to stimulate reparative dentine formation and protect the pulp tissue from further inflammation. Importantly, the reparative dentine structure quality is highly influenced by the signaling molecules contained in the material applied $(2,3)$. The discontinuities and absence of tubular structure in reparative dentine induced by the conventional pulp-capping material (4-13) reduces dentine permeability and does not provide long-term protection of the pulp tissue $(2,3)$.

Dental pulp cells can differentiate into odontoblast-like cells and generate reparative dentine formation in response to damaged dentine. This ability indicates that pulp tissue contains odontogenic progenitor or stem cells that can differentiate into multiple cell lineages, including odontoblasts, osteoblasts, and adipocytes $(14,15)$. However, every cell in an individual carries the same genetic information encoded in their chromosomes (16). Thus, their exposure to specific signaling molecules are essential to control the cell fate decision, proliferation, and differentiation involved in reparative dentin formation $(3,15,17$, 18).

Epigenetic regulation, such as histone modification (19), is one of the ways to turn gene expression on or off by modifying the chromosome structure without changing the DNA sequence (20). This regulation directs the cells into specific lineages $(15,18)$ and involves two enzyme types, histone acetyltransferases (HATs) and histone deacetylases (HDACs), generating histone acetylation and deacetylation, respectively $(19,20)$. Acetylation by HATs is associated with gene activation through nucleosome relaxation. In contrast, deacetylation by HDACs is associated with gene repression through nucleosome compaction (19-21). Eighteen HDACs have been found in humans and are divided into four classes depending on their structural similarities (21-23) with class I (HDAC1, 2, and 3) and class II (HDAC4, 5, and 9) HDACs expressed in dental pulp tissue (24).

Currently, trichostatin A (TSA) and suberoylanilide hydroxamic acid (SAHA or Vorinostat), hydroxamate acid HDAC inhibitors (HDACis) $(20,23,25)$, are the most studied HDACis in the field of odontogenic-induction; however, only SAHA is approved by the United States Food and Drug Administration (FDA) for clinical use in treating cutaneous t-cell lymphoma $(20,25)$. Both are involved in changing the balance between HATs and HDACs by blocking the HDAC active site. This blockade promotes the relaxation of nucleosome structure and facilitates the binding of transcriptional proteins to target genes, resulting in gene transcription $(23,26)$.

Huang et al. (27) reported the direct involvement of TSA in the TGF- $\beta$ pathway that is important in inducing odontogenesis at the early stage of odontoblast-like cell differentiation by upregulating the expression of odontoblast differentiation-related genes, including nuclear factor I C (NFIC), dentin sialophosphoprotein (DSPP), dentin matrix acidic phosphoprotein 1 (DMP1), integrin-binding sialoprotein $(I B S P)$, and bone gamma-carboxyglutamate protein (BGLAP).
TSA also enhances alkaline phosphatase (ALP) activity and mineralization in a dose-dependent manner (28). Moreover, SAHA treatment in an MDPC23 odontoblast-like cell line enhanced their odontoblast differentiation through increased DSPP expression mediated by an increased NFIC expression at the mRNA and protein level, which subsequently induced mineralization in a dose-dependent manner (26). These findings indicated that TSA and SAHA might induce odontoblast differentiation and promote mineralization through increased dentin extracellular matrix production in pulp-derived stem cells (28) and transformed cell lines $(26,27,29)$. However, a comparison of the stimulatory effects on the odontogenicinduction of dental pulp primary cells between TSA and SAHA has not been reported.

The present study aimed to investigate and compare the stimulatory effect of TSA and SAHA in inducing human dental pulp cell (hDPC) odontoblast-like differentiation, mineralized nodule formation, and the expression of odontoblast differentiation-related genes. Therefore, this study may provide valuable information for developing a biocompatible material to induce dentine regeneration.

\section{MATERIALS AND METHODS}

\section{Human Dental Pulp Tissue Preparation}

Four healthy third molars were collected from four patients (18-25 years old) at the Department of Oral Surgery, Faculty of Dentistry, Chulalongkorn University using procedures and informed consent approved by The Human Research Ethics Committee at the Faculty of Dentistry, Chulalongkorn University (HREC-DCU 2020-013). The teeth were immediately rinsed with sterile saline solution and immersed in growth medium (Dulbecco's Modified Eagle Medium (DMEM; \#11960, Gibco ${ }^{\circledR}$ Life Technologies) supplemented with 10\% FBS, 100 units $/ \mathrm{ml}$ penicillin, $100 \mathrm{mg} / \mathrm{ml}$ streptomycin, $2 \mathrm{mM} \mathrm{L}$-glutamine, and $5 \mathrm{mg} / \mathrm{ml}$ amphotericin B). The teeth were maintained at $4^{\circ} \mathrm{C}$ and transferred to the laboratory, and then cleaned with sterile phosphate-buffered saline (PBS) under sterile conditions. Each tooth was held by dental forceps and a scalpel blade was used to remove the periodontal ligament on the root surface.

The tooth crown was broken using a hammer and the dental pulp tissue was gently separated from the tooth fragments. The tissue was rinsed with sterile PBS to remove any tooth chips and prevent infection. It was then cut off into $\sim 1-2 \mathrm{~mm}$ pieces. The human dental pulp cells (hDPCs) were obtained using a tissue outgrowth method.

\section{hDPC Isolation}

Each piece of dental pulp tissue was washed twice with sterile PBS and placed in a $25-\mathrm{mm}$ culture dish containing $1 \mathrm{ml}$ growth medium to allow dental pulp tissue and cell attachment. The culture dishes were incubated at $37^{\circ} \mathrm{C}$ with $5 \% \mathrm{CO}_{2}$, and the medium was changed every $2-3$ days. hDPCs at the third to sixth passage and $80 \%$ confluence were used in the present study. 


\section{HDACi Preparation}

Master stocks of $5 \mathrm{mM}$ TSA (\#58880, Sigma-Aldrich) and SAHA (\#149647, Sigma-Aldrich) in dimethyl sulfoxide (DMSO; \#D2650, Sigma ${ }^{\circledR}$ Life Science) were diluted in PBS before being diluted to 50, 200, 400, and $800 \mathrm{nM}$ TSA; and 0.5, 1, 3, 5, and $10 \mu \mathrm{M}$ SAHA in culture medium.

\section{Cell Viability Assay}

The cell viability of the hDPCS was measured using a 3-(4,5dimethylthiazol-2-yl)-2,5-diphenyl tetrazolium bromide (MTT; \#M6494, Invitrogen, USA) assay. hDPCs were seeded at $2 \times$ $10^{4}$ cells $/ \mathrm{cm}^{2}$ in 96 -well plates containing a growth medium for $24 \mathrm{~h}$ at $37^{\circ} \mathrm{C}$. The cells were incubated in serum-free medium for $4 \mathrm{~h}$ at $37^{\circ} \mathrm{C}$ before being exposed to $50,200,400$, or $800 \mathrm{nM}$ TSA, or $0.5,1,3,5$, or $10 \mu \mathrm{M}$ SAHA in osteogenic medium (DMEM 10\% FBS supplemented with $50 \mu \mathrm{g} / \mathrm{ml}$ ascorbic acid, $5 \mathrm{mM} \beta$-glycerophosphate, and $250 \mu \mathrm{M}$ dexamethasone). The control groups were cultured in growth medium and osteogenic medium without TSA or SAHA.

The MTT powder was diluted in serum-free DMEM without phenol red to a concentration of $0.5 \mathrm{mg} / \mathrm{ml}$. On an experimental day $3(72 \mathrm{~h})$, the medium was removed and the cells were rinsed with PBS. Then $100 \mu \mathrm{l}$ MTT solution was added to each well and incubated at $37^{\circ} \mathrm{C}$ for $30 \mathrm{~min}$. The MTT solution was removed, and the precipitated formazan crystals were dissolved by adding $100 \mu \mathrm{l}$ glycine buffer/DMSO solution at a 1:9 ratio. The plates were placed on an orbital shaker for $45 \mathrm{~min}$ to dissolve the formazan crystals. The absorbance was read within an hour at $570 \mathrm{~nm}$ using a spectrophotometer, and the values were expressed as optical density (OD).

\section{Mineralization Assay}

Human dental pulp cells were seeded at $5 \times 10^{4}$ cells $/ \mathrm{cm}^{2}$ in 24-well plates containing a growth medium at $37^{\circ} \mathrm{C}$. After $24 \mathrm{~h}$ (experimental day-0), the cells were washed with sterile PBS and cultured in osteogenic medium supplemented with 50, 200 , or $400 \mathrm{nM}$ TSA; or 0.5 or $1 \mu \mathrm{M}$ SAHA for 21 days. The control groups were treated with growth medium and osteogenic medium without TSA or SAHA. The medium was changed every 2-3 days.

On experimental day-14 and-21, mineralized nodule formation was examined by staining with Alizarin Red. The cells were washed twice with ice-cold PBS and fixed in ice-cold methanol for $10 \mathrm{~min}$ at room temperature (RT). After completely removing the methanol, the fixed cells were rinsed twice with deionized water and stained by adding $300 \mu \mathrm{l} 1 \%$ alizarin red solution to cover the cell monolayer. The cells were stained for $5 \mathrm{~min}$ at RT, and the mineralized nodule formation was observed using an inverted microscope (Nikon Eclipse TS100, Nikon Instruments Inc., Melville, NY) and quantified with ImageJ Software version 1.51 .

\section{HDAC Activity Assay}

The HDAC activity during TSA and SAHA treatment was measured and analyzed with the fluorometric HDAC Activity Assay kit purchased from abcam ${ }^{\circledR}$ (Abcam, Cambridge, MA). The sample preparation and assay procedure followed the protocol of the manufacturer. Briefly, hDPC cell lysate was collected after $72 \mathrm{~h}$ of $400 \mathrm{nM}$ TSA, $1 \mu \mathrm{M}$ SAHA treatment, or osteogenic medium without HDACis. Then, the lysate was incubated in HDAC assay buffer containing a substrate peptide for $20 \mathrm{~min}$ at $37^{\circ} \mathrm{C}$ in a 96-well black polystyrene microplate (\#27314025, Costar ${ }^{\circledR}$, USA). The reaction was stopped by adding $20 \mu \mathrm{l}$ of stop solution. Then, $5 \mu \mathrm{l}$ of the developer were added to each well of the microtiter plate and incubated for $10 \mathrm{~min}$. The fluorescence intensity was measured with a fluorescence plate reader at $\mathrm{Ex} / \mathrm{Em}=350 / 460 \mathrm{~nm}$ at $10-40 \mathrm{~min}$ and normalized with the blank (i.e., no enzyme control assay containing buffer of the sample). Crude HDAC without HDAC inhibitor was used as a positive control.

\section{Scratch Wound Healing Assay}

The hDPC migration was evaluated using a scratch woundhealing assay. hDPCs at $5 \times 10^{4}$ cells $/ \mathrm{cm}^{2}$ were seeded into 24-well plates and incubated in a growth medium at $37^{\circ} \mathrm{C}$ for $24 \mathrm{~h}$. After a $24 \mathrm{~h}$ serum starvation (counted as experimental day 0), a scratch wound was made through the confluent cell layer using a $200-\mu l$ pipette tip in the middle of each well plate. The cells were washed two times with a serum-free medium to remove cell debris. The hDPCs in the experimental group were incubated with serum-free medium supplemented with $400 \mathrm{nM}$ TSA or $1 \mu \mathrm{M}$ SAHA for $72 \mathrm{~h}$. The control group was treated with a serum-free medium without TSA or SAHA.

Photomicrographs of the scratch were taken using an inverted microscope at $0,24,48$, and $72 \mathrm{~h}$ after the scratch wounding. The plates were marked to register them at each viewing time point. The scratch-wound closure percentage was determined using ImageJ Software version 1.51. The distance between the cells migrated into the wound area and their respective starting points were determined. The distance of wound closure at 24, 48 , and $72 \mathrm{~h}$ postscratch wounding was quantified as a percent of closure and compared with the control group.

\section{RNA Extraction and Real-Time Reverse Transcription-Polymerase Chain Reaction}

Human dental pulp cells at $5 \times 10^{4}$ cells $/ \mathrm{cm}^{2}$ were seeded in six-well plates containing a growth medium for $24 \mathrm{~h}$. After $4 \mathrm{~h}$ of serum starvation at $37^{\circ} \mathrm{C}$, the cells were cultured for 10 days in osteogenic medium without and with $400 \mathrm{nM}$ TSA or $1 \mu \mathrm{M}$ SAHA, with medium changes every 2-3 days. Trizol reagent (\#K00054-0200, Biotechrabbit) was used to obtain total RNA at $24 \mathrm{~h}, 72 \mathrm{~h}, 7$ days, and 10 days postinduction. One microgram of RNA samples was converted to cDNA using reverse transcriptase reaction by ImProm-II Reverse Transcription System (\#A3800, Promega Corporation, Madison, WI). Real-time reverse transcription-polymerase chain reaction (RT-PCR) was performed using CAPITAL ${ }^{\mathrm{TM}}$ qPCR Green Mix HRox (\#BR0501901, BiotecRabbit) with a reaction volume of 20 $\mu \mathrm{l}$ containing $375 \mathrm{nM}$ primers and $1 \mu \mathrm{l}$ RT product. Real-time thermal cycle (CFX96 TouchTM Real-Time PCR System, Bio$\mathrm{Rad})$ was used to perform PCR as follows: initial activation at $95^{\circ} \mathrm{C}$ for $2-3 \mathrm{~min}$, denaturation at $95^{\circ} \mathrm{C}$ for $15 \mathrm{~s}$, and extension at $60-65^{\circ} \mathrm{C}$ for $30 \mathrm{~s}$ for 40 cycles. Bio-Rad CFX Maestro 1.1 manager software was used to analyze the relative gene 
TABLE 1 | Polymerase chain reaction (PCR) primer sequences of target genes.

\begin{tabular}{|c|c|}
\hline Primer & Sequence $\left(5^{\prime}: 3^{\prime}\right)$ \\
\hline \multirow[t]{2}{*}{ VEGFA } & Forward: CCTTGCTGCTCTACCTCCAC \\
\hline & Reverse: ATCTGCATGGTGATGTTGGA \\
\hline \multirow[t]{2}{*}{ CDKN1A } & Forward: TCAGGGTCGAAAACGGCG \\
\hline & Reverse: CCTCTTGGAGAAGATCAGCCG \\
\hline \multirow[t]{2}{*}{ NFIC } & Forward: GACCTGTACCTGGCCTACTTTG \\
\hline & Reverse: CACACCTGACGTGACAAAGCTC \\
\hline \multirow[t]{2}{*}{ COL1A1 } & Forward: GTGCTAAAGGTGCCAATGGT \\
\hline & Reverse: ACCAGGTTCACCGCTGTTAC \\
\hline \multirow[t]{2}{*}{ ALPL } & Forward: CGAGATACAAGCACTCCCACTTC \\
\hline & Reverse: CTGTTCAGCTCGTACTGCATGTC \\
\hline \multirow[t]{2}{*}{ DSPP } & Forward: TCACAAGGGAGAAGGGAATG \\
\hline & Reverse: TGCCATTTGCTGTGATGTIT \\
\hline \multirow[t]{2}{*}{ DMP1 } & Forward: ATGCCTATCACAACAAACC \\
\hline & Reverse: CTCCTTATGTGACAACTGC \\
\hline \multirow[t]{2}{*}{ IBSP } & Forward: ATGGCCTGTGCTTCTCAATG \\
\hline & Reverse: AGGATAAAAGTAGGCATGCTTG \\
\hline \multirow[t]{2}{*}{ BGLAP } & Forward: CTTTGTGTCCAAGCAGGAGG \\
\hline & Reverse: CTGAAAGCCGATGTGGTCAG \\
\hline \multirow[t]{2}{*}{ KLF4 } & Forward: CTGAACAGCAGGGACTGT \\
\hline & Reverse: GTGTGGGTGGCTGTTCTIT \\
\hline \multirow[t]{2}{*}{ Nestin } & Forward: CTG CGG GCT ACT GAA AAG TT \\
\hline & Reverse: AGG CTG AGG GAC ATC TTG AG \\
\hline \multirow[t]{2}{*}{ GAPDH } & Forward: TGAAGGTCGGAGTCAACGGAT \\
\hline & Reverse: TCACACCCATGACGAACATGG \\
\hline
\end{tabular}

expression. The PCR primers used are presented in Table $\mathbf{1}$. All gene expressions were analyzed and normalized to the expression of the housekeeping gene glyceraldehyde 3-phosphate dehydrogenase (GAPDH).

The threshold cycle (CT) from each sample was determined using the real-time cycler software and changed from CT to expression fold change using the Bio-Rad CFX Maestro 1.1 manager software.

\section{ALP Activity}

The hDPC ALP activity during odontoblast-like differentiation was measured at day- $3,-5,-7$, and -10 postinduction. hDPCs at $5 \times 10^{4}$ cells $/ \mathrm{cm}^{2}$ were seeded in 24-well-plates containing growth medium. After $24 \mathrm{~h}$ (at experimental day-0), the cells were exposed to an osteogenic medium with or without $400 \mathrm{nM}$ TSA or $1 \mu \mathrm{M}$ SAHA for 10 days, with medium changes every 2-3 days.

Briefly, the cell lysate from each sample was collected by adding ALP lysis buffer (10 mM Tris- $\mathrm{HCl} \mathrm{pH} \mathrm{10,2} \mathrm{mM} \mathrm{MgCl}_{2}$, and $0.1 \%$ Triton $\mathrm{X}-100)$ and incubated in the substrate mixture of $10 \mu \mathrm{l}$ P-nitrophenyl phosphate (PNPP; N7653, Sigma Life Science) and $100 \mu \mathrm{l}$ of $0.1 \mathrm{M}$ aminopropanol in $2 \mathrm{mM} \mathrm{MgCl}_{2}$, at $37^{\circ} \mathrm{C}$ for $30 \mathrm{~min}$ until it became yellow. The reaction was stopped by adding $900 \mu \mathrm{l}$ of $0.1 \mathrm{M} \mathrm{NaOH}$ to each well. The absorbance of the mixture was measured at $410 \mathrm{~nm}$ wavelength using a microplate reader.
For the total protein assay, the samples were treated in the same manner as in the ALP assay until the samples were frozen. The total protein was analyzed using the BCA protein assay kit (\#3380463, EMD Millipore Corp., USA). After thawing, 200 $\mu \mathrm{l}$ bicinchoninic acid solution was added to the samples and incubated at $37^{\circ} \mathrm{C}$ for $30 \mathrm{~min}$. The absorbance of samples was measured at $562 \mathrm{~nm}$ wavelength using a spectrophotometer, and the amount of total protein was quantified using a standard curve. Furthermore, the ALP activity of the samples was obtained by normalizing the ALP activity product with total protein content compared with the control group.

\section{Statistical Analysis}

The experiments were repeated three to four times. The mean and SD were calculated for each data set. Statistical analysis was performed using the one-way ANOVA and Tukey's HSD posthoc test to compare the experimental groups with control or one experimental group to another. Significant differences were considered at $p<0.05$.

\section{RESULTS}

\section{Effect of TSA and SAHA Treatment on hDPC Viability}

To evaluate the effect of TSA and SAHA treatment on hDPC viability, an MTT assay was performed in an osteogenic medium containing $50,200,400 \mathrm{nM}$, or $800 \mathrm{nM}$ TSA; or $0.5,1,3,5$, or $10 \mu \mathrm{M}$ SAHA for $72 \mathrm{~h}$. Growth medium and osteogenic medium without TSA/SAHA were used to treat the control group. At $72 \mathrm{~h}$ posttreatment, hDPC viability was not influenced by $50 \mathrm{nM}$, $200 \mathrm{nM}$, or $400 \mathrm{nM}$ TSA; or 0.5 and $1 \mu \mathrm{M}$ SAHA treatment compared with the control. In contrast, $800 \mathrm{nM}$ TSA and 3, 5, and $10 \mu \mathrm{M}$ SAHA significantly reduced hDPC viability compared with the control group (Figure 1).

Since 50, 200, and $400 \mathrm{nM}$ TSA; and 0.5 and $1 \mu \mathrm{M}$ SAHA treatment did not impact hDPC viability compared with the control, these concentrations were used in the subsequent experiments.

\section{HDACi Treatment Increased hDPC Mineralized Nodule Formation}

To observe the stimulatory effect and determine the best TSA and SAHA concentration for inducing odontoblast-like differentiation and inducing mineralization in vitro, we stained the hDPC cultures with Alizarin Red on experimental day- 14 and -21 .

On day-14, there was no significant difference in nodule formation in the $50 \mathrm{nM}$ TSA-treated group compared with the osteogenic medium group, indicating that it had a similar effect compared with the osteogenic medium. In contrast, the mineralized nodule formation was significantly elevated in the 200 and $400 \mathrm{nM}$ TSA, and the 0.5 and $1 \mu \mathrm{M}$ SAHA groups compared with the osteogenic medium only group (Figures 2A,B). No mineralized nodules were formed in the growth medium-treated group up to 21 days of 


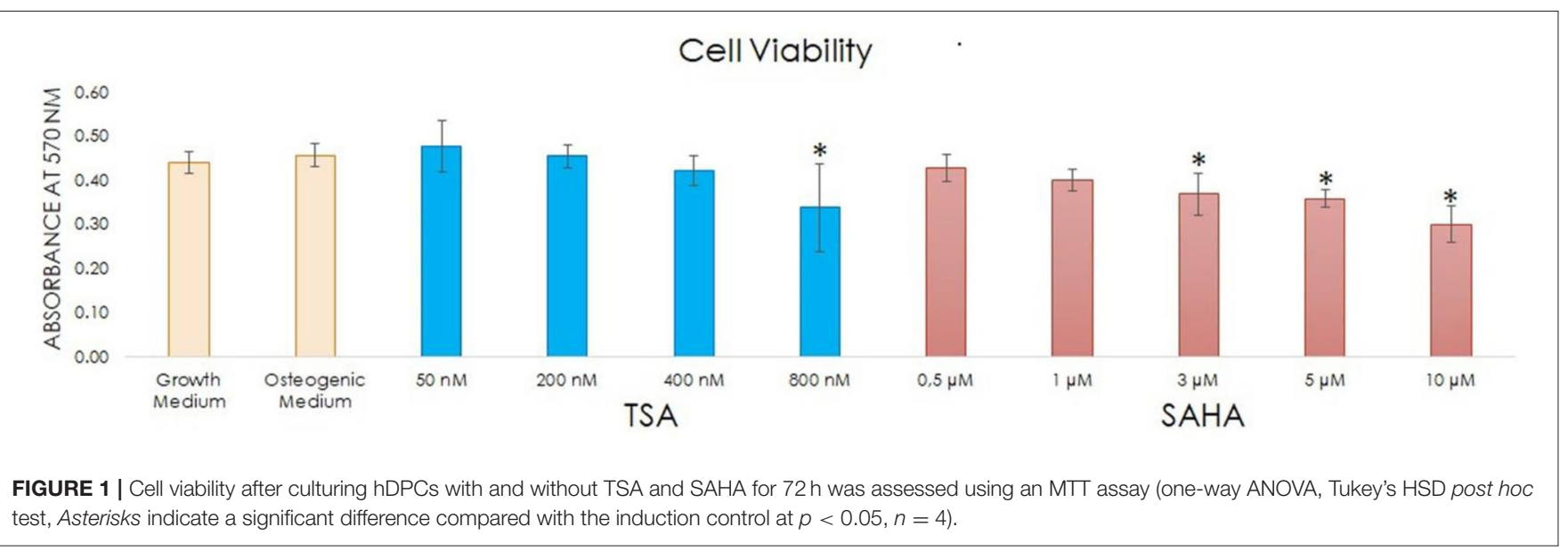

treatment as compared with the osteogenic medium without TSA/SAHA group.

The statistical analysis indicated that $400 \mathrm{nM}$ TSA resulted in higher mineralized nodule formation compared with the osteogenic medium alone. The $1 \mu \mathrm{M}$ SAHA-treated group demonstrated a comparable mineralized nodule formation (Figure 2B). There was no significant difference between the TSA and SAHA groups at 14 days postinduction. Thus, we observed the mineralization for the next 7 days (experimental day-21) using $400 \mathrm{nM}$ TSA and $1 \mu \mathrm{M}$ SAHA, which was assumed to be the optimal mineralization-inducing concentration.

On experimental day-21, the mineralized nodule formations in the $400 \mathrm{nM}$ TSA- and $1 \mu \mathrm{M}$ SAHA-treated group were significantly higher compared with the osteogenic mediumtreated group; however, no significant difference was found between the TSA and SAHA-treated groups (Figures 2C,D). This indicated that $400 \mathrm{nM}$ TSA and $1 \mu \mathrm{M}$ SAHA provide a similar effect in inducing the hDPCs to differentiate into odontoblastlike cells and generate mineralization, as seen in the osteogenic medium-treated group.

\section{Inhibitory Effect of TSA and SAHA in Inhibiting HDAC Activity}

Treatment of $400 \mathrm{nM}$ TSA and $1 \mu \mathrm{M}$ SAHA in hDPCs decreased HDAC activity significantly compared with positive control or osteogenic medium alone after $72 \mathrm{~h}$. In contrast, high fluorescence intensity was found in the osteogenic medium without TSA/SAHA, as seen in positive control (Figure 3). These indicated that TSA and SAHA in samples were active and could inhibit HDAC enzyme activity.

\section{Odontoblast-Related Gene Expression in hDPCs Treated With HDACis}

To confirm the odontogenic effect of TSA and SAHA, the expression of odontoblast differentiation-related genes in hDPCs treated with osteogenic medium containing $400 \mathrm{nM}$ TSA or/and $1 \mu \mathrm{M}$ SAHA was evaluated by RT-qPCR and compared with control cells treated with osteogenic medium without TSA/SAHA.
During the onset of differentiation up to 10 days, the TSA and SAHA-treated groups demonstrated the induction of general mineralization-related gene expression comprising collagen type I alpha 1 chain (COL1A1), alkaline phosphatase $(A L P L), I B S P$, and BGLAP and odontoblast differentiationrelated genes; NFIC, kruppel like factor 4 (KLF4), DMP1, DSPP, and Nestin (Figures 4A-K). TSA and SAHA displayed a similar effect on cyclin-dependent kinase inhibitor 1A (CDKN1A) expression, also known as p21 to induce cell cycle arrest and trigger cell differentiation by inducing downstream gene transcription (30). A significant 6- and 14.9-fold increase in CDKN1A gene expression was found in the TSA- and SAHAtreated group, respectively, $72 \mathrm{~h}$ posttreatment, compared with dependent kinase inhibitor the control. This expression gradually declined at the later stage (day-7 and-10) to 4- and 1.7-fold in the TSA and 13.4- and 7.5-fold in SAHA groups. We found that SAHA significantly upregulated CDKN1A gene expression compared with TSA.

Nuclear factor I C was expressed to initiate the expression of downstream genes related to odontoblast differentiation (31-33), including KLF4 (31). TSA and SAHA treatment similarly increased NFIC and KLF4 expression at the early stage of differentiation. One micromolar SAHA and $400 \mathrm{nM}$ TSA treatment significantly enhanced NFIC expression from 1.7- to 10.6-fold at 24-72 h and 1.5- to 20.4-fold at $72 \mathrm{~h}-7$ days of treatment, respectively, which then gradually decreased at the later stage. KLF4 expression increased 6-fold at $72 \mathrm{~h}$ and 9-fold at 10 days of TSA and SAHA treatment, respectively.

The TSA and SAHA treatments enhanced the hDPC expression of COL1A1, ALPL, DSPP, and IBSP in a similar pattern to that of NFIC and KLF4. The $1 \mu \mathrm{M}$ SAHA group demonstrated a significant 24-, 20.5-, 4.4-, and 7-fold upregulated expression in COL1A1, ALPL, DSPP, and IBSP, respectively, at experimental day-10. Whereas, after 10 days of $400 \mathrm{nM}$ TSA treatment, the expression of COL1A1, ALPL, DSPP, and IBSP were significantly increased by 5.8-, 9-, 2.7-, and 3fold, respectively. Furthermore, a different pattern of vascular endothelial growth factor A (VEGFA) expression, a gene marker for new blood vessel formation (34), was found in HDACis treatment. The $1 \mu \mathrm{M}$ SAHA group significantly increased VEGFA 


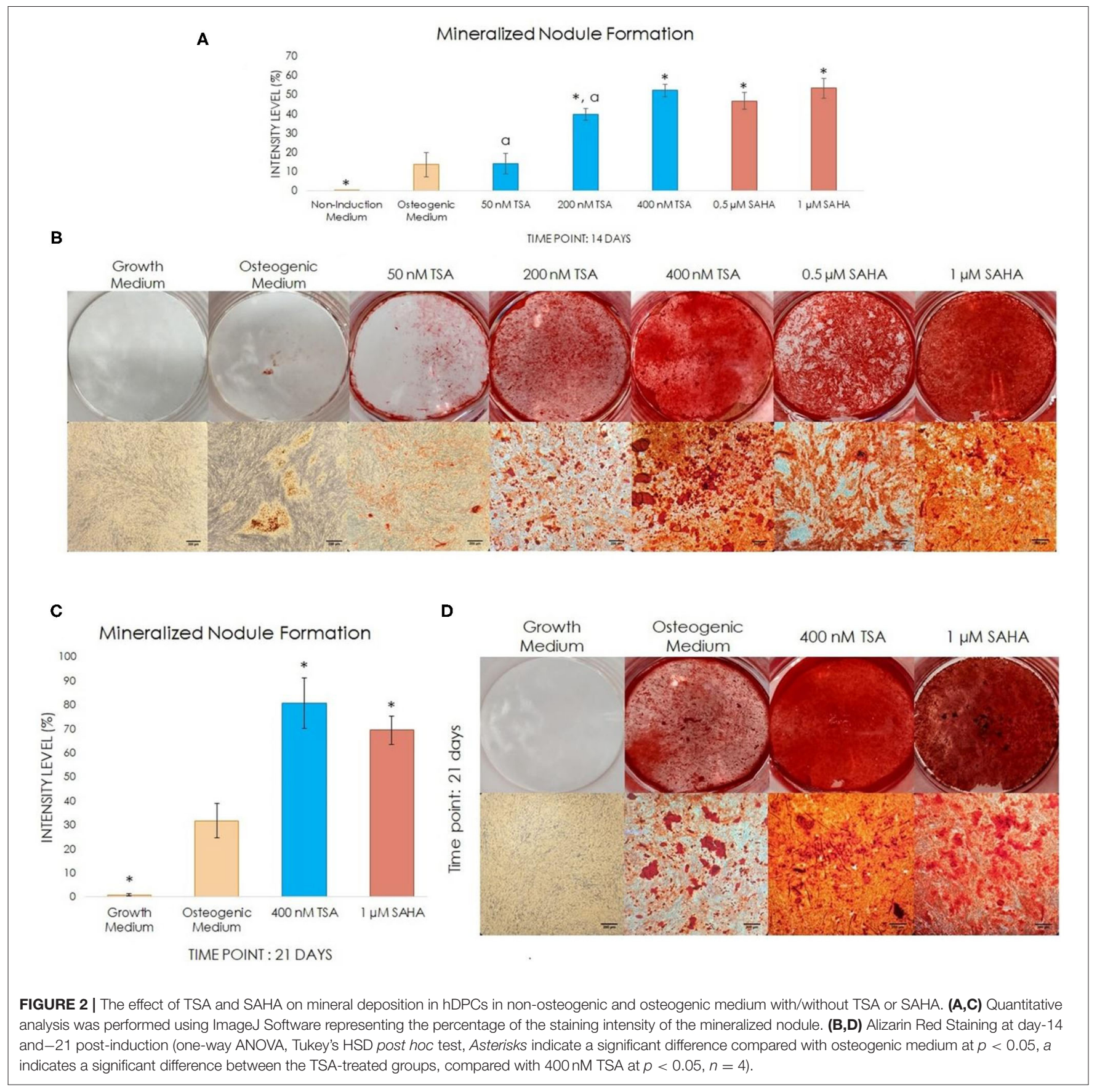

gene expression, upto 36-fold at day-7, 12 times higher when compared with the TSA group. However, its expression was decreased by 7.9 -fold after 10 days of SAHA treatment. In contrast, the $400 \mathrm{nM}$ TSA group demonstrated a significant 12.7fold upregulated expression in VEGFA after $72 \mathrm{~h}$ of treatment. However, its expression dropped to 3 -fold on day-7 and was not detected on day-10. SAHA demonstrated a significantly better effect on COL1A1, ALPL, DSPP, and VEGFA expression on day 10 compared with TSA.
The Nestin expression pattern in the TSA- and SAHA-treated hDPCs observed by RT-qPCR demonstrated a similar pattern to the other gene markers for odontoblast differentiation and mineralization, except for DMP1 and BGLAP. The SAHA group demonstrated a significant increase in Nestin expression from 2.2- to 2.3-fold at 24-72 h, which was 3-fold higher compared with TSA at the $24 \mathrm{~h}$ time-point. In contrast, TSA treatment significantly elevated Nestin expression from 1.5- to 5.5-fold at $72 \mathrm{~h}-7$ days of treatment. In contrast, SAHA maintained the 


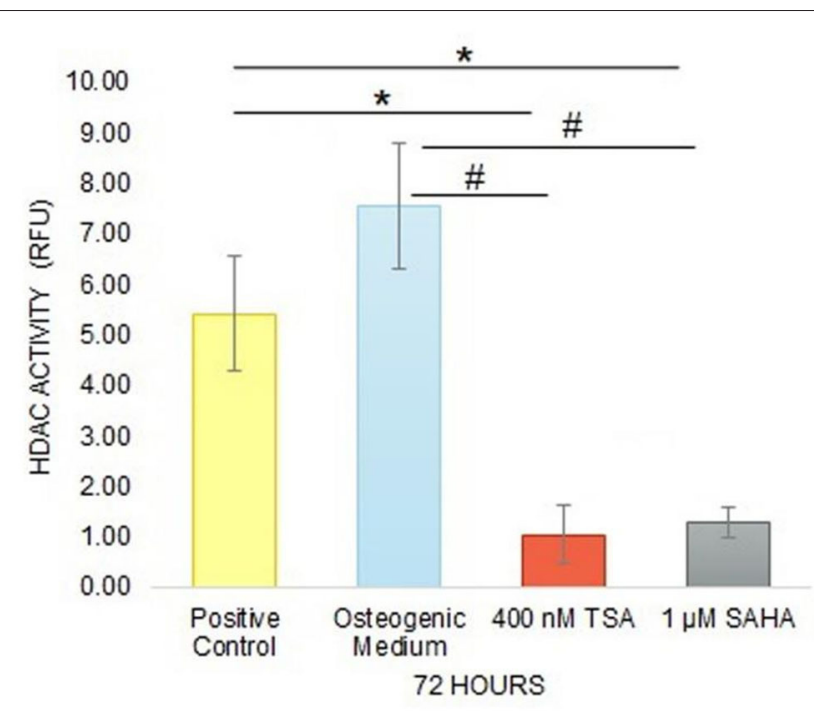

FIGURE 3 | The inhibitory effect of TSA and SAHA in HDAC activity inhibition was evaluated by a HDAC activity assay after $72 \mathrm{~h}$ treatment in hDPC culture (one-way ANOVA, Tukey's HSD post hoc test, Asterisks indicate a significant difference compared with positive control at $p<0.05$, Hashtags indicate a significant difference compared with osteogenic medium at $p<0.05, n=3$ ).

upregulation of Nestin expression up to 4.1-fold after 10 days of treatment compared with the control.

The TSA and SAHA treatments resulted in gradually increased DMP1 gene expression during the onset of differentiation. However, the $400 \mathrm{nM}$ TSA-treated group demonstrated a significantly 8.3-fold increased DMP1 expression on day-10 posttreatment compared with the $1 \mu \mathrm{M}$ SAHA-treated group that demonstrated a 4.7 -fold increase, which was 1.7 -fold lower than the TSA-treated group.

The BGLAP gene, also known as osteocalcin, expression increased 9-fold after 7 days of treatment with $400 \mathrm{nM}$ TSA or $1 \mu \mathrm{M}$ SAHA and increased until 10 days. At this time point, the BGLAP expression in the SAHA-treated group increased 24-fold, which was 1.5 -fold higher than the TSA-treated group.

\section{The Change in ALP Activity During Odontoblast-Like Differentiation in HDACi-Treated hDPCs}

To demonstrate that TSA and SAHA induced ALP activity in osteogenic medium, ALP activity was measured on experimental day-3,-5,-7, and -10 . When treated with $400 \mathrm{nM}$ TSA or $1 \mu \mathrm{M}$ SAHA, the groups demonstrated different patterns in ALP activity. The ALP activity in the $400 \mathrm{nM}$ TSA-treated group significantly increased starting from the early stage until the intermediate stage (experimental day-3 and day-5, respectively) of odontoblast differentiation, compared with the control group. However, the ALP activity decreased at later time points (at experimental day-7 and-10). In contrast, a significant increase in ALP activity was found in the $1 \mu \mathrm{M}$ SAHA-treated group starting from the early stage and continued to increase up to day10 postinduction, compared with the control and TSA-treated groups (Figure 5).

\section{The Stimulatory Effects of TSA and SAHA on Cell Migration}

To evaluate the effect of $400 \mathrm{nM}$ TSA or $1 \mu \mathrm{M}$ SAHA on hDPC migration, a scratch Wound Assay was performed for $72 \mathrm{~h}$ in a serum-free medium. Cell migration is shown as the percentage of wound closure, calculated by dividing the scratch width every $24 \mathrm{~h}$ by the baseline scratch width multiplied by 100 . A significant increase in wound closure was found only at $72 \mathrm{~h}$ postTSA/SAHA treatment compared with control. However, there was no significant difference in scratch closure between the TSA and SAHA groups at that time-point (Figures 6A,B).

\section{DISCUSSION}

In the present study, we demonstrated that the HDAC inhibitors, TSA and SAHA, induced hDPC odontoblast-like cell differentiation without affecting cell viability through increased expression of odontoblast differentiation-related genes and increased ALP activity and mineralized nodule formation. TSA and SAHA, epigenetic-modifying agents, belonging to the hydroxamic acid inhibitor group (25), remove zinc ions from the HDAC active site to inhibit class I and II HDAC activity $(21,23,35)$. This process prevents deacetylation on the lysine residue of the histone core $\mathrm{N}$-terminal tail (36), causing the balance between HATs and HDACs to shift to more acetylated lysine residues (21). Histone acetylation converts the positivelycharged histone core into negatively-charged lysine residues on the histone tails, which relaxes the nucleosome structure $(23,31)$ and provides a binding site for transcriptional proteins to target genes (26) that induce cellular activities, such as proliferation, cell death, differentiation, and cell fate decision $(21,37)$.

In the present study, we found that a high concentration of TSA $(800 \mathrm{nM})$ and SAHA $(3,5$, and $10 \mu \mathrm{M})$ significantly reduced cell viability, suggesting that these concentrations might have a cytotoxic effect on hDPCs. Based on these findings, we hypothesized that TSA and SAHA at concentrations of 50, 200 , and $400 \mathrm{nM}$; and 0.5 and $1 \mu \mathrm{M}$, respectively, would be the appropriate doses for treating the hDPCs in vitro without affecting cell viability.

Our findings correspond to previous studies reporting that $1 \mu \mathrm{M}$ SAHA (26) and 50, 100, 200, and $400 \mathrm{nM}$ TSA $(28,38)$ treatment did not have a cytotoxic effect or cause cell death in either a cell line (MDPC-23) or primary cells (hDPSCs and hPDLCs). However, the high concentration of TSA $(800 \mathrm{nM})$ impacted cell viability (38). In contrast, treating a murine dental pulp-derived cell line (MDPC-23) (26) with 3 and $5 \mu \mathrm{M}$ SAHA did not affect cell viability after $48 \mathrm{~h}$. The low concentration of TSA (25 nM and $400 \mathrm{nM}$ ) was toxic to MDPC-23 cells (29). These disparate results might be caused by differences in cell type, and primary cells vs. transformed cell lines.

After determining which concentrations were not cytotoxic to hDPCs, we evaluated the effect of TSA and SAHA on 


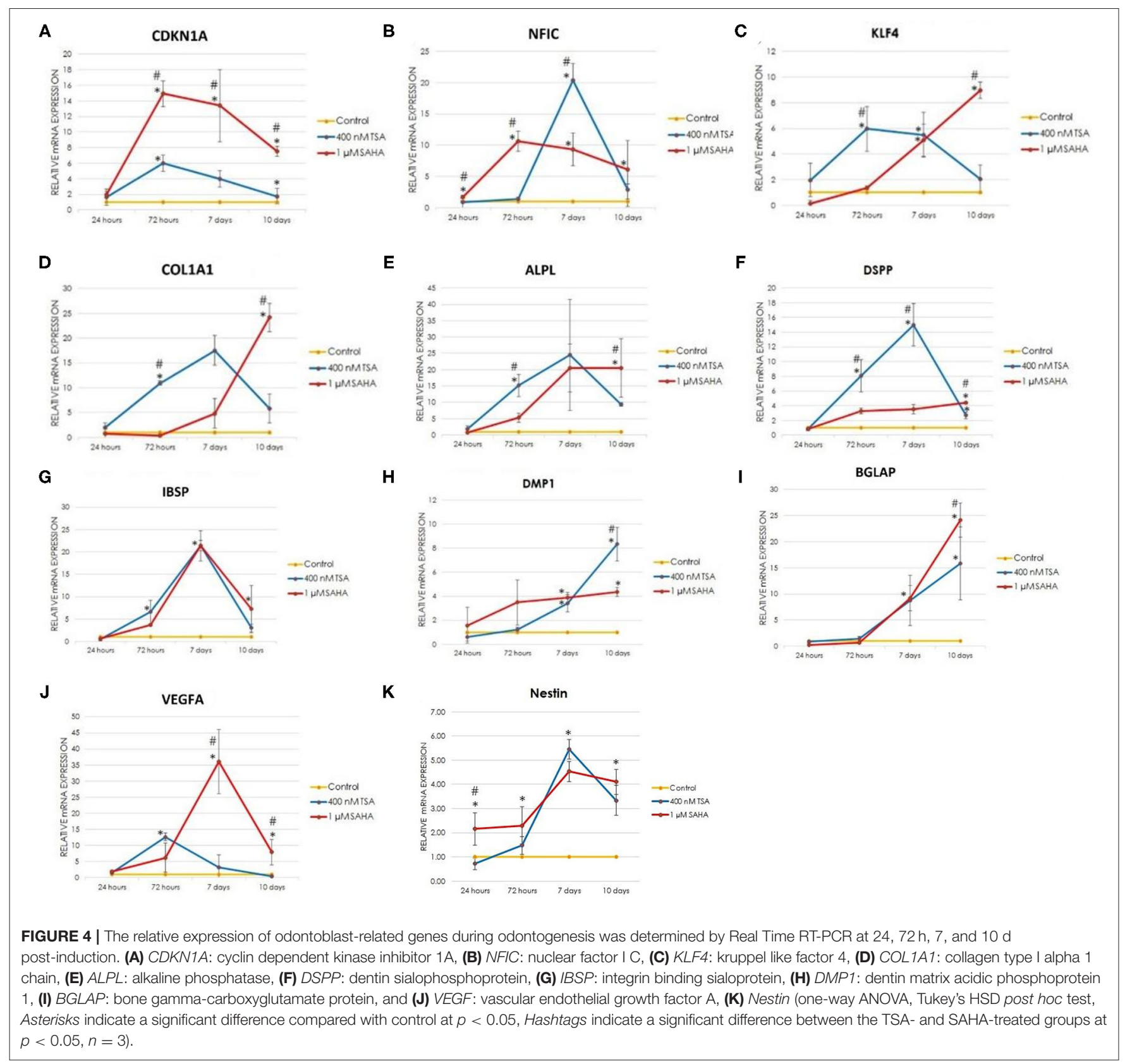

mineralization in osteogenic medium. On experimental day14 , significantly more mineralized nodules were found in the $400 \mathrm{nM}$ TSA- and $1 \mu \mathrm{M}$ SAHA-treated groups, compared with control and other TSA or SAHA groups. However, after 21 days posttreatment, the increased mineralization in the $400 \mathrm{nM}$ TSA and $1 \mu \mathrm{M}$ SAHA groups were not significantly different. These results indicate that both HDACi concentrations had similar odontogenic effects in inducing mineralization, as shown by alizarin red staining quantification, suggesting that HDACi dose selection should be considered to increase odontoblastlike differentiation. Notably, the large mineralized nodules and strong alizarin red staining occurred in the TSA and SAHA concentration range below the concentrations that significantly reduced cell viability. Our results also corresponded with previous studies demonstrating that $400 \mathrm{nM}$ TSA and $1 \mu \mathrm{M}$ SAHA treatment increased mineralization $(26,38)$.

Furthermore, $400 \mathrm{nM}$ TSA and $1 \mu \mathrm{M}$ SAHA treatment in hDPCs culture demonstrated their inhibitory effect in inhibiting HDAC activity after $72 \mathrm{~h}$, which corresponds with HDAC reviews $(23,35)$ reporting that TSA has an inhibitory effect at $\mathrm{nmol} / \mathrm{L}$ concentrations; however, SAHA requires $\mu \mathrm{mol} / \mathrm{L}$ concentrations to inhibit HDAC activity. The reduction of HDAC activity was in line with the increased gene expression, which regulates odontoblast differentiation and maturation.

The present study found significantly elevated CDKN1A expression at the early stage of differentiation, significantly 
elevated expression of the odontoblast-related markers NFIC, KLF4, DMP1, DSPP, and Nestin, and general mineralizedrelated markers COL1A1, ALPL, IBSP, and BGLAP, indicating

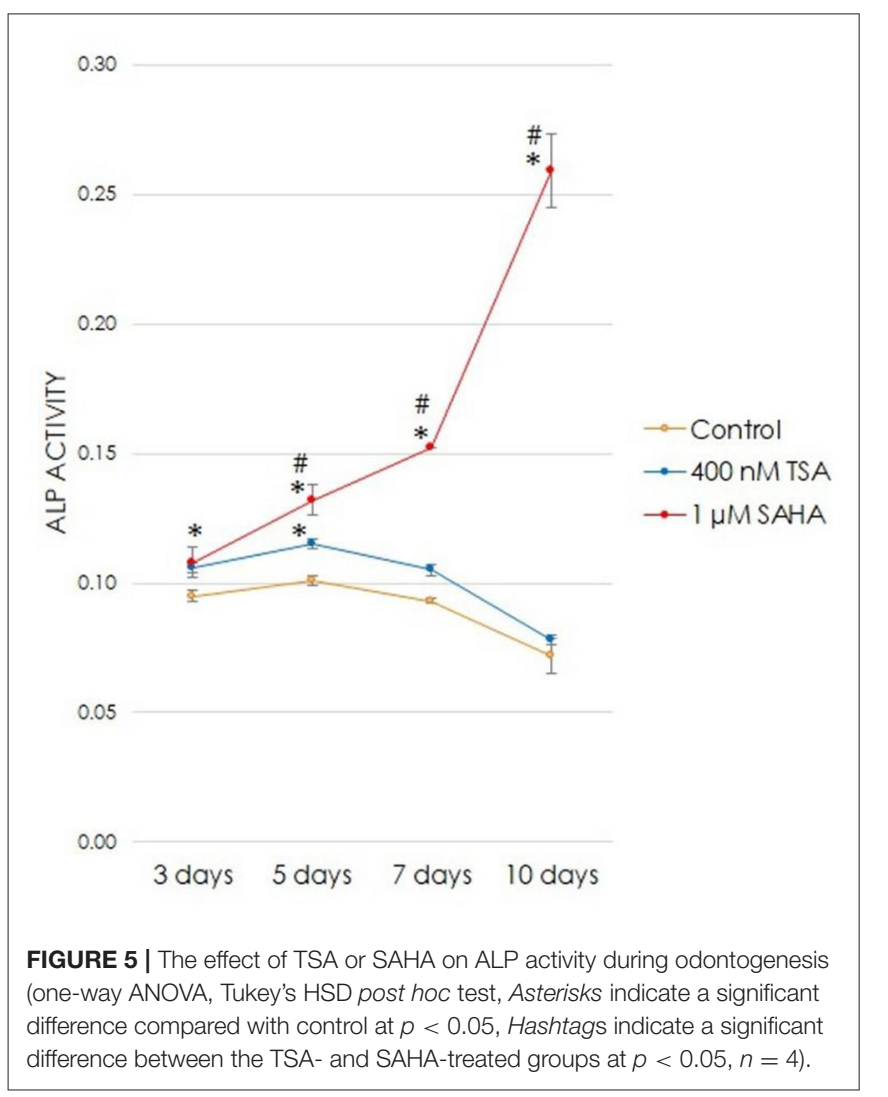

that TSA and SAHA treatment triggered mineralization by inducing cell cycle arrest and differentiation. TSA $(28,29)$ and SAHA (26) treatment have been previously demonstrated to dose-dependently reduce cell growth mediated by increased CDKN1A expression and increased odontoblast differentiation and mineralization.

The present study evaluated the differentiation of dental pulp cells into odontoblast-like cells by detecting a combination of gene markers, such as NF1C, KLF4, DSPP, DMP1, and Nestin. Nestin, an intermediate filament, is involved in tooth development and dentin repair $(39,40)$. Some studies considered Nestin as a marker for differentiated odontoblasts during tooth development and odontoblast-like cells, derived from dental pulp cells, during reparative dentin formation $(40,41)$. Nestin was expressed in the odontoblast processes adjacent to the injury site after cavity preparation (41). The present study also found an increase in Nestin expression during hDPC odontogenesis after TSA and SAHA treatment.

A distinct pattern in COL1A1 and DSPP expression was found during TSA and SAHA treatment. SAHA timedependently induced the expression of COL1A1 and DSPP, which demonstrated a significant 24- and 4.4-fold upregulated expression in COL1A1 and DSPP on day-10, respectively. In contrast to the early onset of induction, a decline in COL1A1 and DSPP expression was found on day-10 of TSA treatment. These findings imply the robust onset of the TSA response. However, SAHA treatment triggered a slower onset, with gradually increased expression of COL1A1 and DSPP up to day-10.

Trichostatin A and SAHA exhibited distinct significant differences in each odontogenic-related expression of the gene at a given time point except for IBSP expression, also known as bone sialoprotein, which was not significantly different over

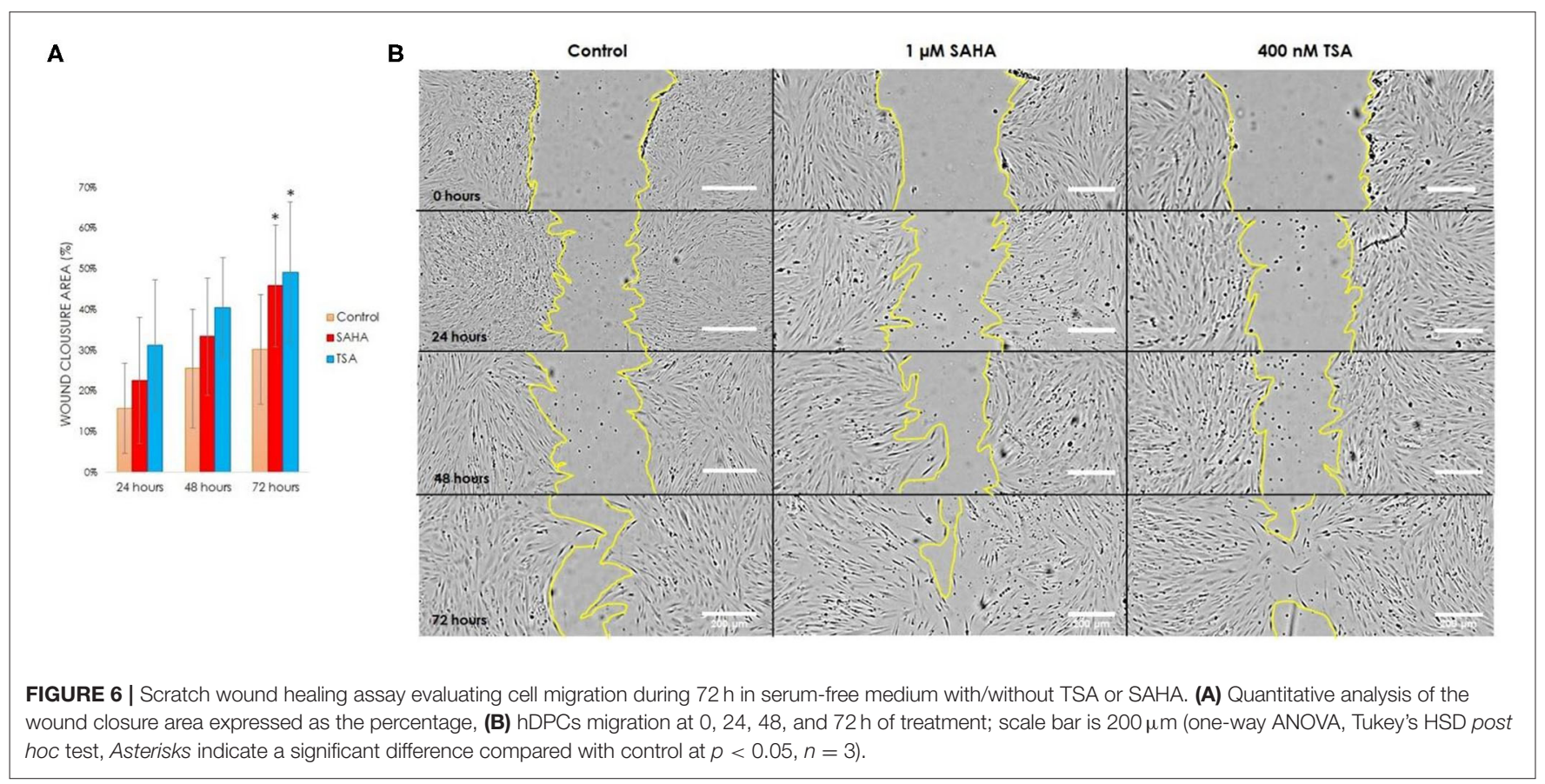


time. In the present study, an increase in the relative expression in most of the genes related to odontoblast differentiation and mineralization was found in the SAHA-treated group compared with the TSA-treated group on day-10 of treatment. These results might partly be due to the slower onset of induction induced by SAHA compared with TSA. TSA induced a more robust increase in the expression of these genes earlier and it declined on day-10. Interestingly, TSA and SAHA gradually elevated DMP1 expression over time and induced hDPC odontoblast differentiation, which corresponds with previous studies $(26,28)$ reporting that TSA treatment in hDPSCs (28) and SAHA treatment in MDPC23 cells (26) increased DMP1 mRNA levels. Further investigation into the possibility that different molecular pathways may be involved for each HDAC inhibitor in inducing cytodifferentiation might be required.

Overall, both HDACis stimulated almost all odontogenicrelated expressions of the genes. Taken together, we hypothesize that TSA and SAHA might have similar odontogenic effects by inhibiting the HDAC active site, leading to histone acetylation and nucleosome relaxation. The HDACi-mediated transcriptional activity induced the expression of target genes related to odontoblast differentiation and generated mineralized nodule formation.

The Nfic-Klf4-Dmp1-Dspp cascade regulates odontoblast differentiation as previously reported. NFIC expression initiates the expression of downstream genes. The binding of NFIC protein to the KLF4 promoter induces its expression, and hence $D M P 1$ gene is expressed via KLF4 protein binding to the DMP1 promoter. $D M P 1$ induces DSPP expression (31-33). DMP1 and $D S P P$ expression induce odontoblast maturation, responsible for dentin extracellular matrix production and secretion, including the collagenous and non-collagenous proteins and mineralization $(17,42)$. In the present study, TSA/SAHA treatment increased NFIC expression, which upregulated KLF4, $A L P, D S P P, D M P 1$, and $B S P$ expression, leading to $\mathrm{hDPC}$ mineralization. A previous study (33) reported that NFIC protein binds to the KLF4 promoter and induced KLF4 gene transcription, which mediated DSPP and DMP1 expression because NFIC knockdown in MDPC23 odontoblast cells reduced KLF4, DMP1, and DSP protein levels. In contrast, NFIC and KLF4 overexpression induced mineralization by upregulating $D S P P$ and $D M P 1$ expression. In contrast, Kwon et al. (26) demonstrated that NFIC protein was directly recruited to the DSPP promoter, leading to increased DSPP expression in MDPC23 cells. NFIC gene knockdown by siRNA reduced the NFIC mRNA level and decreased the expression of odontogenic marker genes, such as DSPP, DMP1, and Nestin.

Alkaline phosphate, encoded by the ALPL gene in humans, induces mineralization by providing phosphate to initiate mineralization (43). The activity of ALP is considered a mineralization indicator $(44,45)$, and high ALP activity has been linked to cells, such as osteoblast and odontoblast cells, forming mineralized tissue (43-45). The present study found differences in ALPL mRNA expression and ALP activity during HDAC inhibitor treatment. At the mRNA level, TSA elevated
ALPL expression from the early onset of differentiation $(72 \mathrm{~h})$ up to 7 days of treatment, which then decreased on day-10. However, at the enzyme activity level, an increase in ALP activity was found up to 5 days of TSA treatment, and a gradual decline was found at the later stage. In contrast, SAHA timedependently enhanced ALPL mRNA expression up to day-10, corresponding with the observed ALP activity. These findings imply that the mRNA level change does not result in a change in enzyme activity. We hypothesize that the ALP protein might undergo modification between the posttranslation and functional stages, influencing its enzyme activity. A review by Robinson (46) explained that environmental factors, such as temperature and $\mathrm{pH}$, might impact enzyme activity changes. Furthermore, a previous study (47) also reported that altered gene expression and enzyme activity correlated with the response of the cell to different physiological conditions. These findings are in accordance with the observed discrepancy in the level of $A L P L$ expression and ALP activity of hDPCs during TSA treatment. We hypothesize that TSA might induce the expression of suppressor genes or generate an environmental change that impacts ALP enzyme activity.

Finally, the observed increase in VEGFA expression and wound closure demonstrated that the inhibitory effect of HDACis is to promote histone acetylation, which might mediate new blood vessel growth (34) and cell migration, respectively. The TSA and SAHA effects on promoting cell migration were observed in serum-free medium to avoid inducing cell proliferation due to serum in the cell culture medium. The significant increase in CDKN1A expression observed at $24-72 \mathrm{~h}$ during TSA and SAHA treatment might explain their effect on cell migration because CDKN1A is associated with cell cycle arrest $(29,48)$.

The HDACis induced cell differentiation in adult stem cell populations (28), primary dental pulp/periodontal ligament cells $(38,48)$, and odontoblast/osteoblast cell lines $(26,29,49)$. Under appropriate conditions and concentrations, HDACis trigger the expression of a series of odontogenic-related genes and mineralization in vitro $(26,28,29,48)$ and in vivo $(28)$. These findings may provide valuable information for developing a potentially biocompatible material to induce reparative dentin formation that can be used as a vital pulp treatment. However, more research is required regarding the HDACi effects on other cell types because HDACis also regulate odontogenesis $(26,28,29,48)$, osteogenesis $(38,49)$, and chondrogenesis (50). The optimal carrier material for HDACis that efficiently delivers an HDACi to the damaged site of dental pulp tissue and releases the HDACi at the appropriate concentration, and also the interaction between an HDACi and the existing restorative material should be considered before clinical use. Moreover, the effect of HDACis on enzyme activity also requires further investigation because TSA and SAHA treatments resulted in different effects on the ALP activity level in the present study.

In conclusion, TSA and SAHA, pan HDAC inhibitors, inhibited HDAC activity and promoted hDPC odontoblastlike cell differentiation and mineralized nodule formation in a dose-dependent manner without interfering with cell 
viability. The increases in gene expression related to odontoblast differentiation and maturation indicate that these two HDACis epigenetically mediate odontoblast-like differentiation and provided further evidence for the odontogenic effects of HDACis. Since HDACis have been approved by the FDA and are used in several clinical treatments, they provide interesting candidates for regenerative biomaterials for dental treatment.

\section{DATA AVAILABILITY STATEMENT}

The original contributions presented in the study are included in the article/supplementary material, further inquiries can be directed to the corresponding author/s.

\section{ETHICS STATEMENT}

The studies involving human participants were reviewed and approved by The Human Research Ethics Committee at the Faculty of Dentistry, Chulalongkorn University (HREC-DCU 2020-013). The patients/participants provided their written informed consent to participate in this study.

\section{REFERENCES}

1. Kearney M, Cooper PR, Smith AJ, Duncan HF. Epigenetic approaches to the treatment of dental pulp inflammation and repair: opportunities and obstacles. Front Genet. (2018) 9:311. doi: 10.3389/fgene.2018. 00311

2. Tziafas D, Smith AJ, Lesot H. Designing new treatment strategies in vital pulp therapy. J Dent. (2000) 28:77-92. doi: 10.1016/S0300-5712(99) 00047-0

3. Tziafas D. Characterization of odontoblast-like cell phenotype and reparative dentin formation in vivo: a comprehensive literature review. J Endod. (2019) 45:241-9. doi: 10.1016/j.joen.2018.12.002

4. Hwang YC, Hwang IN, Oh WM, Park JC, Lee DS, Son HH. Influence of TGF-betal on the expression of BSP, DSP, TGF-betal receptor I and Smad proteins during reparative dentinogenesis. J Mol Histol. (2008) 39:15360. doi: 10.1007/s10735-007-9148-8

5. Cox CF, Sübay RK, Ostro E, Suzuki S, Suzuki SH. Tunnel defects in dentin bridges: their formation following direct pulp capping. Oper Dentisry. (1996) 21:4-11.

6. Faraco IM Jr., Holland R. Response of the pulp of dogs to capping with mineral trioxide aggregate or a calcium hydroxide cement. Dent Traumatol. (2001) 17:163-6. doi: 10.1034/j.1600-9657.2001.170405.x

7. Njeh A, Uzunoglu E, Ardila-Osorio H, Simon S, Berdal A, Kellermann O, et al. Reactionary and reparative dentin formation after pulp capping: hydrogel vs. dycal. Evid Based Endodont. (2016) 1:3. doi: 10.1186/s41121-016-0003-9

8. Murray PE, Garcia-Godoy F. The incidence of pulp healing defects with direct capping materials. Am J Dent. (2006) 19:171-7.

9. Nowicka A, Lagocka R, Lipski M, Parafiniuk M, Grocholewicz K, Sobolewska E, et al. Clinical and histological evaluation of direct pulp capping on human pulp tissue using a dentin adhesive system. Biomed Res Int. (2016) 2016:2591273. doi: 10.1155/2016/2591273

10. Cai S, Zhang W, Tribble G, Chen W. Reactions of human dental pulp cells to capping agents in the presence or absence of bacterial exposure. J Oral Sci. (2017) 59:621-7. doi: 10.2334/josnusd.16-0625

11. Min KS, Park HJ, Lee SK, Park SH, Hong CU, Kim HW, et al. Effect of mineral trioxide aggregate on dentin bridge formation and expression of dentin sialoprotein and heme oxygenase-1 in human dental pulp. J Endod. (2008) 34:666-70. doi: 10.1016/j.joen.2008.03.009

\section{AUTHOR CONTRIBUTIONS}

IS, CL, and RA: conceptualization. IS and RA: methodology, validation, and data curation. IS and TS: software and investigation. IS: formal analysis, writing-original draft preparation, and visualization. CL and RA: resources and supervision. TS, CL, and RA: writing-review and editing. RA: project administration and funding acquisition. All the authors have read and agreed to the published version of the manuscript.

\section{FUNDING}

This research was supported by Faculty Research Grant (DRF64032), Faculty of Dentistry, Chulalongkorn University.

\section{ACKNOWLEDGMENTS}

The authors thank the technical and support staff in the Department of Oral and Maxillofacial Surgery, Department of Microbiology, and Oral Biology Research Center, Faculty of Dentistry, Chulalongkorn University for their assistance. They are grateful to Kevin Tompkins for manuscript revision.

12. Khalil IT, Sarkis T, Naaman A. MM-MTA for direct pulp capping: a histologic comparison with ProRoot MTA in rat molars. J Contemp Dent Pract. (2013) 14:1019-23. doi: 10.5005/jp-journals-10024-1443

13. Rodrigues EM, Cornelio ALG, Mestieri LB, Fuentes ASC, Salles LP, RossaJunior C, et al. Human dental pulp cells response to mineral trioxide aggregate (MTA) and MTA Plus: cytotoxicity and gene expression analysis. Int Endod J. (2017) 50:780-9. doi: 10.1111/iej.12683

14. Gronthos S, Mankani M, Brahim J, Robey PG, Shi S. Postnatal human dental pulp stem cells (DPSCs) in vitro and in vivo. Proc Natl Acad Sci. (2000) 97:13625. doi: 10.1073/pnas.240309797

15. Rodas-Junco BA, Canul-Chan M, Rojas-Herrera RA, De-la-Pena C, Nic-Can GI. Stem cells from dental pulp: what epigenetics can do with your tooth. Front Physiol. (2017) 8:999. doi: 10.3389/fphys.2017.00999

16. Thomas Pollard WE, Lippincott-Schwartz J, Johnson G. Cell Biology. 3rd ed. Philadelphia, PA: Elsevier (2016).

17. Teti G, Salvatore V, Ruggeri A, Manzoli L, Gesi M, Orsini G, et al. In vitro reparative dentin: a biochemical and morphological study. Eur J Histochem. (2013) 57:e23. doi: 10.4081/ejh.2013.e23

18. Lin Y, Zheng L, Fan L, Kuang W, Guo R, Lin J, et al. The epigenetic regulation in tooth development and regeneration. Curr Stem Cell Res Ther. (2018) 13:4-15. doi: 10.2174/1574888X11666161129142525

19. Guidi CJ, Imbalzano AN. Chromatin remodeling and cancer. In: Stein GS, Pardee AB, editors. Cell Cycle and Growth Control. 2nd ed. Hokoben, NJ: John Wiley \& Sons, Inc. (2004). p. 265-95.

20. Duncan HF, Smith AJ, Fleming GJ, Cooper PR. Epigenetic modulation of dental pulp stem cells: implications for regenerative endodontics. Int Endod J. (2016) 49:431-46. doi: 10.1111/iej.12475

21. Huynh NC, Everts V, Ampornaramveth RS. Histone deacetylases and their roles in mineralized tissue regeneration. Bone Rep. (2017) 7:3340. doi: 10.1016/j.bonr.2017.08.001

22. Luo Z, Wang Z, He X, Liu N, Liu B, Sun L, et al. editors. Effects of histone deacetylase inhibitors on regenerative cell responses in human dental pulp cells. Int Endod J. (2018) 1:767-78. doi: 10.1111/iej.12779

23. Hyun-Jung Kim, Bae S-C. Histone deacetylase inhibitors: molecular mechanisms of action and clinical trials as anti-cancer drugs. Am J Transl Res. (2011) 3:166-79.

24. Klinz FJ, Korkmaz Y, Bloch W, Raab WH, Addicks K. Histone deacetylases 2 and 9 are coexpressed and nuclear localized in 
human molar odontoblasts in vivo. Histochem Cell Biol. (2012) 137:697-702. doi: 10.1007/s00418-012-0920-9

25. Eckschlager T, Plch J, Stiborova M, Hrabeta J. Histone deacetylase inhibitors as anticancer drugs. Int J Mol Sci. (2017) 18:1414. doi: 10.3390/ijms18071414

26. Kwon A, Park HJ, Baek K, Lee HL, Park JC, Woo KM, et al. Suberoylanilide hydroxamic acid enhances odontoblast differentiation. J Dent Res. (2012) 91:506-12. doi: 10.1177/0022034512443367

27. Huang W, Zhao S, Ammanamanchi S, Brattain M, Venkatasubbarao K, Freeman JW. Trichostatin A induces transforming growth factor beta type II receptor promoter activity and acetylation of $\mathrm{Sp} 1$ by recruitment of PCAF/p300 to a Sp1.NF-Y complex. J Biol Chem. (2005) 280:1004754. doi: $10.1074 /$ jbc.M408680200

28. Jin H, Park JY, Choi H, Choung PH. HDAC inhibitor trichostatin A promotes proliferation and odontoblast differentiation of human dental pulp stem cells. Tissue Eng Part A. (2013) 19:613-24. doi: 10.1089/ten.tea.2012.0163

29. Duncan HF, Smith AJ, Fleming GJ, Cooper PR. Histone deacetylase inhibitors induced differentiation and accelerated mineralization of pulp-derived cells. $J$ Endod. (2012) 38:339-45. doi: 10.1016/j.joen.2011.12.014

30. Ouellet S, Vigneault F, Lessard M, Leclerc S, Drouin R, Guerin SL. Transcriptional regulation of the cyclin-dependent kinase inhibitor 1A (p21) gene by NFI in proliferating human cells. Nucleic Acids Res. (2006) 34:647287. doi: $10.1093 / \mathrm{nar} / \mathrm{gkl} 861$

31. Tao H, Lin H, Sun Z, Pei F, Zhang J, Chen S, et al. Klf4 promotes dentinogenesis and odontoblastic differentiation via modulation of TGF-beta signaling pathway and interaction with histone acetylation.J Bone Miner Res. (2019) 34:1502-16. doi: 10.1002/jbmr.3716

32. Roh SY, Park JC. The role of nuclear factor I-C in tooth and bone development. J Korean Assoc Oral Maxillofac Surg. (2017) 43:63-9. doi: 10.5125/jkaoms.2017.43.2.63

33. Lee HK, Lee DS, Park SJ, Cho KH, Bae HS, Park JC. Nuclear factor I-C (NFIC) regulates dentin sialophosphoprotein (DSPP) and E-cadherin via control of Kruppel-like factor 4 (KLF4) during dentinogenesis. J Biol Chem. (2014) 289:28225-36. doi: 10.1074/jbc.M114.568691

34. Saghiri MA, Asatourian A, Sorenson CM, Sheibani N. Role of angiogenesis in endodontics: contributions of stem cells and proangiogenic and antiangiogenic factors to dental pulp regeneration. J Endod. (2015) 41:797803. doi: 10.1016/j.joen.2014.12.019

35. Dokmanovic M, Clarke C, Marks PA. Histone deacetylase inhibitors: overview and perspectives. Mol Cancer Res. (2007) 5:981-9. doi: 10.1158/1541-7786.MCR-07-0324

36. Duncan HF, Smith AJ, Fleming GJ, Cooper PR. HDACi: cellular effects, opportunities for restorative dentistry. J Dent Res. (2011) 90:137788. doi: 10.1177/0022034511406919

37. Duncan HF, Smith AJ, Fleming GJ, Partridge NC, Shimizu E, Moran GP, et al. The histone-deacetylase-inhibitor suberoylanilide hydroxamic acid promotes dental pulp repair mechanisms through modulation of matrix metalloproteinase-13 activity. J Cell Physiol. (2016) 231:798816. doi: $10.1002 / j c p .25128$

38. Huynh NC, Everts V, Pavasant P, Ampornaramveth RS. Inhibition of histone deacetylases enhances the osteogenic differentiation of human periodontal ligament cells. J Cell Biochem. (2016) 117:1384-95. doi: 10.1002/jcb.25429

39. Fujita S, Hideshima K, Ikeda T. Nestin expression in odontoblasts and odontogenic ectomesenchymal tissue of odontogenic tumours. J Clin Pathol. (2006) 59:240-5. doi: 10.1136/jcp.2004.025403
40. Quispe-Salcedo A, Ida-Yonemochi H, Nakatomi M, Ohshima H. Expression patterns of nestin and dentin sialoprotein during dentinogenesis in mice. Biomed Res. (2012) 33:119-32. doi: 10.2220/biomedres.33.119

41. About I, Laurent-Maquin D, Lendahl U, Mitsiadis TA. Nestin expression in embryonic and adult human teeth under normal and pathological conditions. Am J Pathol. (2000) 157:287-95. doi: 10.1016/S0002-9440(10)64539-7

42. Yamakoshi Y. Dentinogenesis and dentin sialophosphoprotein (DSPP). J Oral Biosci. (2009) 51:134. doi: 10.1016/S1349-0079(09)80021-2

43. Woltgens JH, Lyaruu DM, Bronckers AL, Bervoets TJ, Van Duin M. Biomineralization during early stages of the developing tooth in vitro with special reference to secretory stage of amelogenesis. Int J Dev Biol. (1995) 39:203-12.

44. Kuru L, Griffiths GS, Petrie A, Olsen I. Alkaline phosphatase activity is up regulated in regenerating human periodontal cells. J Periodont Res. (1999) 34:123-7. doi: 10.1111/j.1600-0765.1999.tb02231.x

45. Golub EE, Boesze-Battaglia K. The role of alkaline phosphatase in mineralization. Curr Opin Orthopaed. (2007) 18:4448. doi: 10.1097/BCO.0b013e3282630851

46. Robinson PK. Enzymes: principles and biotechnological applications. Essays Biochem. (2015) 59:1-41. doi: 10.1042/bse0590001

47. Glanemann C, Loos A, Gorret N, Willis LB, O’Brien XM, Lessard PA, et al. Disparity between changes in mRNA abundance and enzyme activity in Corynebacterium glutamicum: implications for DNA microarray analysis. Appl Microbiol Biotechnol. (2003) 61:61-8. doi: 10.1007/s00253-0021191-5

48. Duncan HF, Smith AJ, Fleming GJ, Cooper PR. Histone deacetylase inhibitors epigenetically promote reparative events in primary dental pulp cells. Exp Cell Res. (2013) 319:1534-43. doi: 10.1016/j.yexcr.2013.02.022

49. Dudakovic A, Evans JM, Li Y, Middha S, McGee-Lawrence ME, van Wijnen AJ, et al. Histone deacetylase inhibition promotes osteoblast maturation by altering the histone $\mathrm{H} 4$ epigenome and reduces Akt phosphorylation. J Biol Chem. (2013) 288:28783-91. doi: 10.1074/jbc.M113.489732

50. Lee J, Im GI. Effects of trichostatin $\mathrm{A}$ on the chondrogenesis from human mesenchymal stem cells. Tissue Eng Regen Med. (2017) 14:40310. doi: 10.1007/s13770-017-0041-6

Conflict of Interest: The authors declare that the research was conducted in the absence of any commercial or financial relationships that could be construed as a potential conflict of interest.

Publisher's Note: All claims expressed in this article are solely those of the authors and do not necessarily represent those of their affiliated organizations, or those of the publisher, the editors and the reviewers. Any product that may be evaluated in this article, or claim that may be made by its manufacturer, is not guaranteed or endorsed by the publisher.

Copyright (๑) 2021 Sulistyowati, Sukpaita, Limjeerajarus and Ampornaramveth. This is an open-access article distributed under the terms of the Creative Commons Attribution License (CC BY). The use, distribution or reproduction in other forums is permitted, provided the original author(s) and the copyright owner(s) are credited and that the original publication in this journal is cited, in accordance with accepted academic practice. No use, distribution or reproduction is permitted which does not comply with these terms. 